\title{
Countertransference in Swedish psychotherapists: testing the factor structure of the Therapist Response Questionnaire
}

\author{
Johan Berg, ${ }^{1}$ Lars-Gunnar Lundh, ${ }^{2}$ Fredrik Falkenström ${ }^{3}$ \\ ${ }^{1}$ School of Social Work, Lund University; ${ }^{2}$ Department of Psychology, Lund University; ${ }^{3}$ Department of Behavioural Sciences and \\ Learning, Linköping University, Sweden
}

\begin{abstract}
Questionnaires need testing of reliability and factor structure before clinical use or research in new languages or cultures. The aim of this study was to evaluate the Therapist Response Questionnaire (TRQ) in Sweden compared to corresponding factor analyses in USA and Italy. A national sample of psychotherapists $(\mathrm{N}=242)$ registered their countertransference with a single client using TRQ. The data were analyzed with confirmatory factor analysis (CFA) to test factor structures from previous studies, and exploratory factor analysis (EFA). The CFA did not verify the factor structure from the previous studies. The EFA extracted seven factors as the best solution: Helpless/Inadequate, Overwhelmed/Disorganized, Hostile/Angry, Parental/Protective, Disengaged, Special/Overinvolved, Sexualized. Analysis of convergent validity indicated that five of these could be considered equivalent to factors in the previous studies, and the remaining two were conceptually related to corresponding factors. Even though the factor structure was not confirmed by the CFA, the concordance was large, indicating a reliable self-report instrument with promising validity for measurement of complex aspects of countertransference. Common countertransference themes can inform psychotherapy supervision and education, give feedback to the therapist, and lay ground for a taxonomy for therapist reactions and feelings.
\end{abstract}

Key words: Countertransference; Therapist Response Questionnaire; Confirmatory factor analysis; Exploratory factor analysis.

Correspondence: Johan Berg, School of Social Work, Lund University, Box 23, 22100 Lund, Sweden.

E-mail: johan.berg@soch.lu.se

Citation: Berg, J., Lundh, L.-G., \& Falkenström, F. (2019). Countertransference in Swedish psychotherapists: testing the factor structure of the Therapist Response Questionnaire. Research in Psychotherapy: Psychopathology, Process and Outcome, 22(1), 99-112. doi: 10.4081/ripppo.2019.331

Acknowledgments: the authors would like to thank the participating psychotherapists.

Contributions: JB, LGL, translation of questionnaire; JB, data collection and exploratory factor analysis; FF, confirmatory factor analysis; JB, LGL, FF, manuscript writing and reviewing.

Conflict of interest: the authors declare no potential conflict of interest.

Funding: the first author was supported by Bertil Wennborgs stiftelse.

Received for publication: 25 August 2018.

Revision received: 2 November 2018.

Accepted for publication: 2 November 2018.

This work is licensed under a Creative Commons Attribution NonCommercial 4.0 License (CC BY-NC 4.0).

CCopyright J. Berg et al., 2019

Licensee PAGEPress, Italy

Research in Psychotherapy:

Psychopathology, Process and Outcome 2019; 22:99-112

doi:10.4081/ripppo.2019.331

\section{Introduction}

Although a therapist's way of relating to a patient is by definition informed by a certain theory and method (techniques, procedures, stance), it will inevitably also involve a number of more personal, non-methodological aspects. For example, the therapist will respond emotionally to the patient, and these feelings may be expressed in various ways, voluntarily or involuntarily. In the psychotherapy literature, these feelings, the corresponding thoughts and the way they are expressed have been referred to as countertransference since Freud (1910) first introduced this term. Even if the concept has its origin in psychoanalysis, its importance is in no way confined to psychoanalytic or psychodynamic treatments, and is relevant to all kinds of psychotherapeutic orientations, however unevenly described and studied.

Freud $(1910,1915)$ famously warned against acting on countertransference, in the sense that the psychoanalyst must handle the impulses to act in accordance with the transference (i.e. transference-love) in neutral way. In his Recommendations to Physicians Practicing PsychoAnalysis, Freud (1912) advised the psychoanalyst to put aside feelings for example of sympathy for the patient, and to become unaffected by own therapeutic ambitions to achieve. This definition of countertransference, based on Freud's technical papers, as the psychoanalyst's own unconscious and conflict-based reaction to the patient is often labeled as the classical view.

Since Freud, the concept of countertransference has 
diversified and received different meanings, ranging from the problematic contribution of the therapist due to own unresolved issues, to a wider view including the whole range of feelings, actions and impulses in the psychotherapist towards the particular client. Winnicott (1949), for example, argued that there was an objective form of countertransference in which the therapist responds to the patient in the same way that others in general tend to do, implying that countertransference can contain useful information in understanding relational patterns. Heimann (1950) suggested that the therapist's emotional responses to the patient is not simply an obstacle or hindrance due to unresolved issues in the therapist's past, but the therapist's most important instrument for understanding the patient and the relationship between them. This wider definition of countertransference is often referred to as the totalistic view but has also been questioned because it could be tempting to blame the patient for the therapist's own difficulties (Gabbard, 2001; Hayes, 2004). More recently, Gabbard $(1995,2001)$ has argued that different views of countertransference in psychoanalysis have approached a common ground, and that "psychoanalytic theorists from diverse persuasions have converged on the idea that, to some extent, countertransference is always a joint creation involving contributions from both clinician and patient" (Gabbard, 2001, p. 989).

From a pantheoretical view Hayes, Gelso and Hummel (2011) have suggested a third definition, an integrative conception of countertransference, comprising aspects of both the classical and totalistic view. According to this definition, countertransference is defined closer to the classical definition as reactions due to therapist's personal vulnerabilities, but not restricted to unconscious issues or to transferential pulls on action. At the same time the integrative view recognizes the potential usefulness in the therapeutic process by understanding the source inside the therapist and its implications in the psychotherapy. Therefore, the integrative view advocates the management of countertransference.

Hayes (2004) has developed a structural theory of countertransference, breaking it down to five concepts. The origins are the intrapsychic conflicts and unresolved issues in the therapist. Triggers are specific events in the therapy that merges with the therapist's own conflicts, creating reactions. It is the combination of origins and triggers that causes countertransference. The manifestations are the variety of reactions, emotional, cognitive, behavioral, that the therapist experiences and displays. The effects are the consequences of these reactions, and finally the management is the therapist ability to handle and minimize the potential negative effect on the therapeutic process.

In a meta-analysis, the management of countertransference was shown to correlate significantly with outcome in psychotherapy (Hayes et al., 2011). The ability to manage countertransference may further be expected to benefit from a thorough understanding of the varied nature of these manifestations, based on a detailed exploration and categorization of these ways of responding. Although it has traditionally been customary in clinical literature to make a global distinction between positive and negative countertransference, loosely defined as a general liking or sympathy for the patient $v s$ a variety of adverse behavior and unpleasant feelings, or as positive $v s$ negative effects of countertransference on psychotherapy outcome, recent empirical studies tend to offer a substantially more complex and nuanced portrait of the nature of these processes.

Countertransference has been studied thru various methods and designs, typically in case studies, in interviews, using external observer's or supervisor's assessment of therapist countertransference behavior, or in therapist self-report questionnaires (Colli \& Ferri, 2015; Hayes et al., 2011). By using self-report instruments and factor analysis, a number of different categories of countertransference manifestations have been identified, mainly based on the totalistic definition of countertransference as the integrative approach to countertransference is difficult to access with self-report measures.

One type of self-report instrument that has been used is various forms of Feeling Word Checklist (FWC), starting with Whyte, Constantopoulos and Bevans' (1982) 30item version. FWC consists of a set of words and the therapist is asked to rate if he or she felt helpful, happy, angry, enthusiastic, anxious, etc., when talking to the patient, with the intention to capture the emotional countertransference. Later researchers have constructed and used versions with 24 items (Lindqvist et al., 2017; Ulberg et al., 2013), 48 items (Holmqvist, 2001; Holmqvist, Hansjons-Gustafsson, \& Gustafsson, 2002) or 58 items (Dahl, Røssberg, Bøgwald, Gabbard, \& Høglend, 2012; Røssberg, Hoffart, \& Friis, 2003). When these various versions of FWCs have been subjected to factor analysis, the resulting number of factors has varied from three (Hoffart \& Friis, 2000) over four (Dahl et al., 2012; Holmqvist et al., 2002; Lindqvist et al., 2017) to seven (Holmqvist \& Armelius, 1994; Røssberg et al., 2003). Among the possible reasons for this diversity in results is not only that the number or selection of items and the response format has varied (e.g., some versions but not others using Likert scales), but also that different patient group or settings (e.g. inpatient units) have been involved.

The FWC is typically administered after a session and asks for the therapist's feelings during that specific session. In contrast to the FWC, the Therapist Response Questionnaire (TRQ; Zittel Conklin \& Westen, 2003) focuses on recurrent themes in countertransference, and not only on feelings in the most recent session. Also, the TRQ is more comprehensive (79 items) and includes a wider scope of countertransference reactions, comprising both feelings (e.g., "I feel nurturant toward him/her") and behavior (e.g., "I disclose my feelings with him/her more than with other patients"). Factor analyzing the TRQ in an American sample of psychotherapists, Betan, Heim, Zittel Conklin and 
Westen (2005) identified eight factors: overwhelmed/disorganized, helpless/inadequate, positive, special/overinvolved, sexualized, disengaged, parental/protective, and criticized/mistreated. Replicating this study in an Italian sample, Tanzilli, Colli, Del Corno and Lingiardi (2016) arrived at a nine-factor solution, basically replicating the factors from Betan et al.'s study, with the addition of a hostile/angry factor. There were however some differences between the two studies in what items loaded on these factors (see Tanzilli et al., 2016, for a summary of the differences), and no confirmatory factor analysis was reported comparing the two studies.

Even if most literature in this area has a psychoanalytic background and has focused on the idiosyncratic aspects of countertransference, some of this research has aimed at studying common themes in countertransference through the use of self-report instruments for the psychotherapist, regardless of psychotherapeutic school. Interestingly, when Betan et al. (2005) eliminated all psychodynamically or psychoanalytically oriented therapists $(40 \%)$ from their sample and carried out a factor analysis on the remaining sample of therapists, basically the same factor structure was reproduced as in the complete sample. Tanzilli et al. (2016) showed a similar result when they excluded the psychodynamic clinicians and found no difference in correlations between factor scores and patients' personality pathology. It can be argued that the factor structure of TRQ is not affected by therapist's theoretical base (Tanzilli et al., 2016), further supporting Hayes (2004) notion of countertransference as a transtheoretical concept.

The TRQ has also been used to map different therapist reactions to patients' personality disorder (Betan et al., 2005; Colli, Tanzilli, Dimaggio, \& Lingiardi, 2014; Gazzillo et al., 2015; Tanzilli, Lingiardi, \& Hilsenroth, 2018), and to study countertransference with patients with eating disorder (Satir, Thompson-Brenner, Boisseau, \& Crisafulli, 2009; Colli et al., 2015) and suicidal behavior (Yaseen et al., 2013). Monitoring countertransference also has potentials as a feedback instrument for the therapist, and to capture negative or harmful reactions in the mind of the therapist, as for example reactions connected to burnout or compassion fatigue (e.g., Kanter, 2007). Countertransference is also considered a key aspect in psychodynamic diagnosis (Lingiardi \& McWilliams, 2017; McWilliams, 2011; PDM Task Force, 2006), especially regarding personality disorders. As the emphasis on countertransference is dependent on different psychotherapeutic traditions and educational settings with potential national variations, there is a need for testing its factor structure in new languages and cultural settings before transporting it for clinical use and research in these settings. The present study had this more limited purpose. At the same time, it may be argued that the attempt to develop a taxonomy of countertransference responses is an important goal in itself, because it may contribute to an increased clarification of important dimensions of the therapeutic relationship, promote countertransference awareness, help to identify interpersonal patterns from the therapist view and counteract potentially adverse effects of different forms of countertransference.

The first purpose of this study therefore was to use the TRQ to analyze patterns in countertransference responses in a population of psychotherapists in Sweden, and to see if the factor structure from the American and Italian studies could be replicated. Our hypothesis was that the factor structure from one of these studies would be replicated. Secondly, the aim was to use background data about the therapists to describe and compare countertransference in the sample.

\section{Materials and Methods}

\section{Sampling}

Clinicians working with psychotherapy in Sweden were asked to participate in a validation study. In order to receive as many forms as possible and a wide range of therapists, the group was sampled through various channels. This included asking four national organizations for psychotherapists to use their email lists (two for cognitive-behavioral therapies (CBT), one for psychodynamic therapies and one for psychoanalysts, in total approx. 3000 emails), Facebook groups for psychologists, universities training psychotherapists and their psychotherapy supervisors. There is presumably a considerable overlap between the various sampling channels. This sampling strategy (convenience sampling) was used to collect a large enough sample for factor analysis in the relatively limited community of psychotherapists in Sweden. A web form was used to collect the information from the individual clinician. The clinicians were not payed to participate, and all were anonymous.

\section{Inclusion/exclusion criteria}

To be able to compare the results with two previous factor analyses (Betan et al., 2005; Tanzilli et al., 2016), the same criteria for inclusion and exclusion for both psychotherapist and patient were used as in the previous studies: The psychotherapist were required to have at least 3 years post-licensure experience with psychotherapy, and at least 10 weekly hours of psychotherapeutic practice. The patient had to be at least 18 years old and to have been in psychotherapy for at least 8 sessions. The psychotherapist was instructed to choose the last patient in the previous week that met the criteria, and who was non-psychotic and not treated with drug therapy for psychotic symptoms.

\section{Participants}

In total 273 psychotherapists filled in the form online, and 31 were excluded due to exclusion criteria (1 due to patient diagnosis of psychosis, 3 patients were just 17 
years old, 18 therapies had lasted less than 8 sessions, 12 therapists worked with psychotherapy less than 10 hours per week; 3 protocols were excluded for more than one reason), resulting in 242 valid forms used in the factor analysis and further calculations.

We report a summary of the sample background variables along with the corresponding data from Betan et al. (2005) and Tanzilli et al. (2016), and available data about licensed psychotherapists in Sweden from the national registry (Table 1).

The patients were diagnosed by the therapists as following: 112 (46\%) had an anxiety, stress-related or somatoform disorder (ICD-10 F40-F49), 81 (33\%) had a mood disorder (ICD-10 F30-39), 30 (12\%) had a personality disorder (ICD-10 F60-69), 2 (1\%) had mental or behavioral disorder due to psychoactive substance use (ICD-10 F10F19), 2 (1\%) had an eating disorder (ICD-10 F50), and 15 $(6 \%)$ had other (ICD-10 F99) or no stated psychiatric diagnosis (e.g., personal therapy with therapist in training). The therapists were instructed to state a diagnosis using ICD-10, DSM-IV, DSM-5 or in plain language, and the diagnosis was then transformed to an appropriate ICD-10 category by the first author. ICD-10 was preferred to DSM-5 as there was no control of formal diagnostical criteria necessary for DSM-5, and therefore the ICD-10's descriptive diagnostical categories were considered more accurate.

Due to the sampling procedure (presumably partly overlapping e-mail lists), it was not possible to estimate response rate. No patients were directly involved in this study. No identifying data about patients was collected.

Table 1. Sample characteristics, background variables.

\begin{tabular}{|c|c|c|c|c|}
\hline & $\begin{array}{l}\text { Present study } \\
\quad(N=242)\end{array}$ & $\begin{array}{l}\text { National } \\
\text { statistics* }\end{array}$ & $\begin{array}{c}\text { Betan et al., } 2005 \\
(N=181)\end{array}$ & $\begin{array}{c}\text { Tanzilli et al., } 2016 \\
(N=332)\end{array}$ \\
\hline \multicolumn{5}{|l|}{ Therapists } \\
\hline \multicolumn{5}{|l|}{ Gender, $n(\%)$} \\
\hline Female & $180(74 \%)$ & $3577(73 \%)$ & $75(41 \%)$ & $180(54 \%)$ \\
\hline Male & $61(25 \%)$ & $1313(27 \%)$ & $106(59 \%)$ & $152(46 \%)$ \\
\hline Other & $1(0.4 \%)$ & & - & - \\
\hline Age, $M(S D)$ & $52(10.2)$ & $a_{1}^{2}$ & & $47(9.8)$ \\
\hline \multicolumn{5}{|l|}{ Basic professional training, $n(\%)$} \\
\hline Psychologist & $121(50 \%)$ & $1610(55 \%)$ & $141(78 \%)$ & $(70 \%)$ \\
\hline Social worker & $69(29 \%)$ & $880(30 \%)$ & - & - \\
\hline Nurse & $14(6 \%)$ & $211(7 \%)$ & - & - \\
\hline Psychiatrist & $6(2 \%)$ & $204(7 \%)$ & $40(22 \%)$ & $(30 \%)$ \\
\hline Other & $32(13 \%)$ & & - & - \\
\hline Therapy workload, average weekly hours (SD) & $22(8.8)$ & & - & $16(3.9)$ \\
\hline \multicolumn{5}{|l|}{ Setting, $n(\%)$} \\
\hline Private practice & $123(51 \%)$ & & $145(80 \%)$ & $(65 \%)$ \\
\hline Other work setting & $119(49 \%)$ & & & \\
\hline \multicolumn{5}{|l|}{ Theoretical orientation, $n(\%)$} \\
\hline Cognitive-behavioral & $97(40 \%)$ & & $37(20 \%)$ & $163(49 \%)$ \\
\hline Psychodynamic & $125(52 \%)$ & & $73(40 \%)$ & $169(51 \%)$ \\
\hline Eclectic & $16(7 \%)$ & & $55(30 \%)$ & - \\
\hline Family therapy & $4(2 \%)$ & & - & - \\
\hline Experience as therapist, years (SD) & $15(9.5)$ & & - & $10(4.5)$ \\
\hline \multicolumn{5}{|l|}{ Patients } \\
\hline \multicolumn{5}{|l|}{ Gender, $n(\%)$} \\
\hline Female & $178(74 \%)$ & & Approx. 50\% & $174(52 \%)$ \\
\hline Male & $62(26 \%)$ & & Approx. $50 \%$ & $158(48 \%)$ \\
\hline Other & $2(1 \%)$ & & - & - \\
\hline Age, $M(S D)$ & $36(11.3)$ & & $40(13.4)$ & $40(5.2)$ \\
\hline \multicolumn{5}{|l|}{ Therapies } \\
\hline Length of treatment, sessions (SD) & $35(73.6)$ & & $19(30.0)$ & Approx. 16-20 (0.9) \\
\hline
\end{tabular}

*Statistics about licensed psychotherapists in Sweden from The National Board of Health and Welfare (Socialstyrelsen), 2016. 


\section{Measures}

\section{Therapist Response Questionnaire}

The Therapist Response Questionnaire (TRQ; Zittel Conklin \& Westen, 2003) is a 79-item questionnaire designed to measure various reactions and responses a psychotherapist can have or feel (i.e., countertransference) during psychotherapy with a particular patient. The questionnaire is filled out by the psychotherapist alone, without the participation of the patient. The items in TRQ are selected to capture different responses, ranging from relatively simple feelings (e.g., "I feel angry at him/her") to more complex situations (e.g., "I have to stop myself from saying or doing something aggressive or critical"), from relatively common feelings (e.g., "I feel like I understand him/her") to presumably rarer situations (e.g., "I tell $\mathrm{him} /$ her I love him/her"). The items are written in everyday language, without explicit theoretical assumptions, easily recognizable for the clinician. Each item is rated on a 5-point Likert scale, ranging from 1 (not true) to 5 (very true). The translation into Swedish was done by the first author, and the translation was verified with a back-translation by the second author, and further inspected by a senior language teacher. The factors in TRQ have previously been shown to have good internal consistency (Cronbach's alpha $.75 \leq \alpha \leq .90$ ). Convergent validity was tested by analyzing correlations with the factors in the two previous studies, showing high intercorrelation $(.78 \leq r \leq .98)$. Criterion validity was tested by distinguishing patients' personality disorder through the therapists' response in TRQ (Betan et al., 2005; Tanzilli et al., 2016).

\section{Demographic information}

In addition to TRQ, the clinician stated some basic demographic data about themselves (age, gender), working site (e.g., private practice, employed or other), basic therapeutic education (e.g., psychologist, psychiatrist, social worker, nurse), principal theoretical orientation (e.g., cognitive-behavioral, psychodynamic, eclectic), number of years of psychotherapeutic experience after basic therapeutic training, and number of hours with weekly psychotherapeutic practice with patients. They further reported age and gender of the patient, number of sessions in the therapy, and diagnosis.

\section{Statistical analysis}

The statistical analysis was made in two steps. First a confirmatory factor analysis (CFA) was carried out, using Mplus version 7.3 (Muthén \& Muthén, 1998-2017), to test the factor structures demonstrated in Betan et al. (2005) and in Tanzilli et al. (2016). Goodness of fit was evaluated using the $\chi^{2}$ statistic, where a nonsignificant value represents an acceptable fit, in combination with the comparative fit index (CFI), the root mean square error of approximation (RMSEA), and the standardized root mean square residual (SRMR). Acceptable fit standards of the latter are $\mathrm{CFI} \geq .90$, RMSEA $\leq .08$, and $\mathrm{SRMR} \leq .10(\mathrm{Hu} \&$ Bentler, 1999).

The second step was an exploratory factor analysis (EFA) using SPSS Version 23 (Mac), also used for other statistical analyses. As suggested by Tanzilli et al. (2016) Principal axis factoring (PAF) was used as the extraction method. As the factors were presumed to be correlated, to some extent, oblique rotation was used (Promax, as recommended by Matsunaga, 2010). The preliminary number of factors was decided by scree plot and parallel analysis, and then EFA was also calculated with extraction of \pm 2 factors to decide on interpretability and clarity of structure. Items loading $\geq|.40|$ on a factor were included in the respective factor, whereas items with a cross loading $\geq|.30|$ were excluded from the factor; 25 items were not included in the factor scores due to low factor loadings (the following items were excluded due to low factor loadings: 1 . I am very hopeful about the gains s/he is making or will likely make in treatment. 5. I wish I had never taken him/her on as a patient. 11. I don't trust what s/he's telling me. 18. I feel depressed in sessions with him/her. 19. I look forward to sessions with him/her. 24. I feel guilty about my feelings toward him/her. 26. I feel overwhelmed by his/her strong emotions. 32 . His/her sexual feelings toward me make me anxious or uncomfortable. 34. I feel I am walking on eggshells around him/her, afraid that if I say the wrong thing s/he will explode, fall apart, or walk out. 35. S/he frightens me. 38. I feel interchangeable - that I could be anyone to him/her. 40. I feel like I understand him/her. 44. I feel like I'm being mean or cruel to him/her. 45 . I have trouble relating to the feelings $\mathrm{s} / \mathrm{he}$ expresses. 46. I feel mistreated or abused by him/her. 50 . I tell him/her I love him/her. 51. I feel overwhelmed by his/her needs. 53. I feel pleased or satisfied after sessions with him/her. 58. I think or fantasize about ending the treatment. 59. I feel like my hands have been tied or that I have been put in an impossible bind. 62. I feel repulsed by him/her. 67 . I end sessions overtime with him/her more than with my other patients. 75 . I watch the clock with $\mathrm{him} /$ her more than with my other patients. 76. I self-disclose more about my personal life with him/her than with my other patients. 77. More than with most patients, I feel like I've been pulled into things that I didn't realize until after the session was over), and 10 items were excluded because of cross loadings over the limit (the following items were excluded due to cross loading over limit: 3. I find it exciting working with him/her. 10. I feel confused in sessions with him/her. 20. I feel envious of, or competitive with him/her. 22. I feel frustrated in sessions with him/her. 29. S/he tends to stir up strong feelings in me. 39. I have to stop myself from saying or doing something aggressive or critical. 48. I lose my temper with him/her. 60 . When checking my phone messages, I feel anxiety or dread that there will be one from him/her. 66. I worry about him/her after sessions more than other patients. 74 . $\mathrm{S} /$ he is one of my favorite patients). The factor structure 
was also checked with Maximum Likelihood estimation with similar results, indicating a stable factor structure (Tabachnick \& Fidell, 2014).

As a third step, the background variables were compared with a summed score for all TRQ items, using correlations, t-tests and analysis of variance (ANOVA).

\section{Results}

The CFA failed to confirm the factor structure from Betan et al. $(2005), \chi^{2}(1349)=2736.81, \mathrm{P}<.001 ; \mathrm{CFI}=.706$; RMSEA $=.066 ;$ SRMR $=.084$. Nor did the confirmatory factor analysis verify the factor structure from Tanzilli et al. (2016), $\chi^{2}(1793)=3730.94, \mathrm{P}<.001$; $\mathrm{CFI}=.678$; RMSEA $=$ .068 ; SRMR $=.091$.

As the next step, the data were subjected to EFA. The Kaiser-Meyer-Olkin measure (KMO) was .86 indicating the data were sufficient for EFA. The Bartlett's test of sphericity $\chi^{2}(3081)=10308.31, \mathrm{P}<.001$ showed that there were patterned relationships between the items, also suggesting suitability for EFA. The scree plot indicated seven factors, and this was also confirmed by parallel analysis. To check the interpretability $7 \pm 2$ factors were extracted. This also confirmed seven factors as the clearest solution with at least four items with sufficient loading on every single factor to maximize the factors' stability. The solution accounted for $45 \%$ of the variance which is lower than the $69 \%$ in Betan et al. (2005) and 58\% in Tanzilli et al. (2016).

Table 2 shows the factor loadings after rotation for the items belonging to each factor. The factor labels are maintained from Betan et al. (2005) and Tanzilli et al. (2016).

Factor 1: Helpless/Inadequate. This factor refers to negative emotions, in the form of feeling incompetent, inadequate, unsuccessful, unappreciated, helpless and hopeless with the patient. There are feelings of worry, anxiety, dread and guilt in the contact with the patient.

Factor 2: Overwhelmed/Disorganized. This factor has mainly behavioral contents and refers to the therapist finding him/herself being controlling with the patient, setting firm boundaries for the patient, and feeling used or manipulated. The therapist also regrets things he/she has said to the patient, and notices that he/she delays returning phone calls. The theme is that the therapist experiences a need to control or limit the patient, supposedly triggered by therapist's feelings of being overwhelmed by the patient's needs or disorganized in the interaction.

Factor 3: Hostile/Angry. This is an emotional factor, which refers to the therapist feeling annoyed, irritated, angry, dismissed, devalued, or criticized by the patient. The therapist can even feel enraged at the patient or tell the patient that he/she is angry with him/her. He/she sometimes dislikes the patient.

Factor 4: Parental/Protective. This is the only emotional factor with positive content. The items loading on this factor shows the therapist as having warm, nurturant, protective, parental feelings towards the patient. He/she likes the patient very much, and the patient makes the therapist feel good about him/herself. The therapist feels compassion for the patient and anger at other people in the patient's life.

Factor 5: Disengaged. This is an emotional factor, mirroring a quality of distance or lack of interest. The therapist feels bored, disengaged and uninterested with the patient.

Factor 6: Special/Overinvolved. This is a behavioral factor, referring to the therapist as stretching the limits in different ways, performing more acts, being more involved, and setting other boundaries for him/herself with this particular patient. The therapist discloses his/her own feelings with the patient (more than with other patients), discusses the patient with supervisor or colleagues more than other patients, begin sessions later, does more for the patient, calls the patient between sessions, or talks about the patient with a partner. The theme is that the therapist is pushing the boundaries, supposedly triggered by feelings of being overinvolved or preoccupied with the patient.

Factor 7: Sexualized. The therapist feels sexual attraction and tension, noticing him/herself being flirtatious or could imagine being friends with the patient. The correlation with all other factors was low (Table 3). As this four-item factor had low internal consistency (Cronbach's $\alpha=.43$ ), the included items were further analyzed. One item ("If s/he were not my patient, I could imagine being friends with him/her.") stood out as lowering the reliability and this item also had the lowest factor loading. When this item was excluded, the reliability improved (Cronbach's $\alpha=69$ ). Therefore, this item was excluded from further use in factor scores.

Table 3 shows the factor correlation matrix. The intercorrelations are rather high among all but two factors, leaving Parental/Protective and Sexualized with lower correlations with other factors. Factors scores were computed by calculating the mean of the items that loaded significantly on the factor. This method is usually considered adequate for EFA (DiStefano, Zhu, \& Mîndrilă, 2009; Tabachnick \& Fidell, 2014) and has the advantage over other methods in making it easy to transfer results to other studies. Table 4 sums the descriptive statistics for the factor-based scales and shows them to have acceptable psychometric properties. Only the positive factor Parental/ Protective is normally distributed, whereas the others are positively skewed, as would be expected (i.e. it would be surprising if single factors with potentially problematic contents had neutral skewness).

A total TRQ score was calculated by summing all 79 variables, and Table 4 also shows the psychometric properties for this variable. This TRQ total score was supposed to capture the general level of self-reported countertransference.

Convergent validity of the TRQ was examined by correlating factor scores from the seven factors found in the 
Table 2. Factor Structure of the Therapist Response Questionnaire, Swedish translation $(N=242)$.

Factors and items, with factor loadings

Factor 1: Helpless/Inadequate

I feel incompetent or inadequate working with him/her. (36)

I feel I am failing to help him/her or I worry that I won't be able to help him/her. (31)

I think s/he might do better with another therapist or in a different kind of therapy. (54)

I feel anxious working with him/her. (30)

I dread sessions with him/her. (13)

I feel guilty when $\mathrm{s} /$ he is distressed or deteriorates, as if I must be somehow responsible. (28)

I feel less successful helping him/her than other patients. (68)

I feel hopeless working with him/her. (52)

I feel unappreciated by him/her. (63)

Factor 2: Overwhelmed/Disorganized

I find myself being controlling with him/her. (37)

I feel pushed to set very firm limits with him/her. (55)

I return his/her phone calls less promptly than I do with my other patients. (70)

I feel used or manipulated by him/her. (33)

I regret things I have said to him/her. (43)

\section{9}

.54

.51

.49

.48

Factor 3: Hostile/Angry

I feel annoyed in sessions with him/her. (8)

I feel angry at him/her. (15)

I feel dismissed or devalued. (6)

At times I dislike him/her. (2)

I get enraged at him/her. (27)

I feel criticized by him/her. (12)

I tell him/her I'm angry at him/her. (41)

Factor 4: Parental/Protective

I have warm, almost parental feelings toward him/her. (64)

I feel nurturant toward him/her. (47)

I feel like I want to protect him/her. (42)

I like him/her very much. (65)

S/he makes me feel good about myself. (23)

I feel angry at people in his/her life. (14)

I feel compassion for him/her. (4)

I feel sad in sessions with him/her. (49)

I wish I could give him/her what others never could. (21)

Factor 5: Disengaged

I feel bored in sessions with him/her. (16)

My mind often wanders to things other than what s/he is talking about. (25)

I don't feel fully engaged in sessions with him/her. (9)

I feel resentful working with him/her. (57)

Factor 6: Special/Overinvolved

I disclose my feelings with him/her more than with other patients. (71)

I find myself discussing him/her more with colleagues or supervisors than my other patients. (73)

I begin sessions late with him/her more than with my other patients. (78)

I do things for him/her, or go the extra mile for him/her, in ways that I don't do for other patients. (69)

I call him/her between sessions more than my other patients. (72)

I talk about him/her with my spouse or significant other more than my other patients. (79)

Factor 7: Sexualized

I feel sexually attracted to him/her. (17)

I feel sexual tension in the room. (61)

I find myself being flirtatious with him/her. (56)

If s/he were not my patient, I could imagine being friends with him/her. (7)*

*This item was excluded from the factor score calculation due to reducing the factor's reliability. 
current study with factor scores based on the eight factors from the American version (Table 5) and the nine factors from the Italian version (Table 6). This confirmed the strong similarity between the factors Helpless/Inadequate, Parental/Protective, Disengaged, and Sexualized in the current and both of the previous studies $(r \geq .90)$, and between the factor Hostile/Angry in the present study and the corresponding factor in the Italian study $(r \geq .90)$. The factors Overwhelmed/Disorganized and Special/Overinvolved also had strong correlations $(r>.70)$ with the same named factors from the two previous studies, but somewhat lower than the above-mentioned factors. The Overwhelmed/Disorganized factor differed from corresponding factors in earlier studies in that the items included in the Swedish study had mainly behavioral contents whereas the American or Italian counterparts mixed behavioral and emotional items.

Table 7 summarizes the main comparisons between the background variables and the factor scores/the TRQ total score. Some background variables were significantly associated with TRQ scores. Therapists who were psychodynamically oriented, male, younger, working in other settings (for example mental health clinics) than private practice and in longer therapies reported more countertransference, compared to female therapists, CBT therapists, older or in private practice. On the other hand, patients' age or gender, or therapist caseload did not seem to affect TRQ. Nor did the therapists' basic professional education affect TRQ, that is, there were no differences between psychologists, social workers, nurses, psychiatrists.

Table 3. Factor correlation matrix $(N=\mathbf{2 4 2})$.

\begin{tabular}{|c|c|c|c|c|c|c|c|}
\hline Factors & 1 & 2 & 3 & 4 & 5 & 6 & 7 \\
\hline 1. Helpless/Inadequate & - & & & & & & \\
\hline 2. Overwhelmed/Disorganized & $.50^{* *}$ & - & & & & & \\
\hline 3. Hostile/Angry & $.52^{* *}$ & $.50^{* *}$ & - & & & & \\
\hline 4. Parental/Protective & $.24^{* *}$ & .10 & $.19^{* *}$ & - & & & \\
\hline 5. Disengaged & $.49^{* *}$ & $.38^{* *}$ & $.50^{* *}$ & .07 & - & & \\
\hline 6. Special/Overinvolved & $.44^{* *}$ & $.39^{* *}$ & $.40^{* *}$ & $.38^{* *}$ & $.23^{* *}$ & - & \\
\hline 7. Sexualized & .06 & $.26^{* *}$ & $.23^{* *}$ & .11 & $.17^{* *}$ & $.35^{* *}$ & - \\
\hline
\end{tabular}

$* * \mathrm{P}<.01$.

Table 4. Descriptive statistics for the factor scores and a Therapist Response Questionnaire (TRQ) total score ( $N=\mathbf{2 4 2})$.

\begin{tabular}{lcccccc}
\hline Factors & No. of items & $\boldsymbol{M}$ & $\boldsymbol{S D}$ & Skewness & Kurtosis & Cronbach's alpha \\
\hline 1. Helpless/Inadequate & 9 & 1.74 & .66 & 1.00 & .50 & .87 \\
\hline 2. Overwhelmed/Disorganized & 5 & 1.37 & .49 & 2.06 & 5.53 & .71 \\
\hline 3. Hostile/Angry & 7 & 1.62 & .64 & 1.12 & .84 & .83 \\
\hline 4. Parental/Protective & 9 & 2.82 & .71 & .25 & -.16 & .79 \\
\hline 5. Disengaged & 4 & 1.47 & .57 & 1.48 & 2.14 & .70 \\
\hline 6. Special/Overinvolved & 6 & 1.36 & .45 & 1.79 & 3.46 & .68 \\
\hline 7. Sexualized & 3 & 1.16 & .37 & 2.65 & 7.23 & .69 \\
\hline Sum of all items (TRQ total score) & 79 & 143.29 & 26.86 & .89 & .55 & .93 \\
\hline
\end{tabular}

Table 5. Intercorrelations for factor scores between the current factor structure and the Betan et al. (2005) factor structure $(N=\mathbf{2 4 2})$.

\begin{tabular}{|c|c|c|c|c|c|c|c|c|}
\hline \multirow[b]{2}{*}{ Factors } & \multicolumn{8}{|c|}{ Subscales based on Betan et al.'s (2005) factor structure } \\
\hline & $\begin{array}{l}\text { Overwhelmed/ } \\
\text { Disorganized }\end{array}$ & $\begin{array}{c}\text { Helpless/ } \\
\text { Inadequate }\end{array}$ & Positive & $\begin{array}{c}\text { Special/ } \\
\text { Overinvolved }\end{array}$ & Sexualized & Disengaged & $\begin{array}{l}\text { Parental/ } \\
\text { Protective }\end{array}$ & $\begin{array}{l}\text { Criticized/ } \\
\text { Mistreated }\end{array}$ \\
\hline 1. Helpless/Inadequate & 0.65 & 0.96 & -0.31 & 0.56 & 0.07 & 0.53 & 0.28 & 0.67 \\
\hline 2. Overwhelmed/Disorganized & 0.78 & 0.53 & -0.19 & 0.37 & 0.26 & 0.48 & 0.14 & 0.58 \\
\hline 3. Hostile/Angry & 0.68 & 0.55 & -0.13 & 0.35 & 0.24 & 0.68 & 0.20 & 0.77 \\
\hline 4. Parental/Protective & 0.14 & 0.29 & 0.44 & 0.46 & 0.13 & 0.08 & 0.93 & 0.19 \\
\hline 5. Disengaged & 0.57 & 0.51 & -0.28 & 0.30 & 0.16 & 0.90 & 0.06 & 0.49 \\
\hline 6. Special/Overinvolved & 0.51 & 0.47 & 0.15 & 0.81 & 0.36 & 0.28 & 0.39 & 0.43 \\
\hline 7. Sexualized & 0.22 & 0.07 & 0.17 & 0.22 & 0.98 & 0.18 & 0.14 & 0.21 \\
\hline
\end{tabular}




\section{Discussion}

Even if the CFA did not confirm the factor structure from earlier studies from the USA (Betan et al., 2005) or Italy (Tanzilli et al., 2016), there was large overlap when comparing the Swedish factor structure to factors found in those studies for the majority of the factors. Four of the factors (Helpless/Inadequate, Parental/Protective, Disengaged and Sexualized) clearly replicated factors from both the American and the Italian study, and a fifth of the factors (Hostile/Angry) replicated a factor from the Italian study. Two of the factors, Overwhelmed/Disorganized and Special/Overinvolved, had somewhat lower correlations and fewer overlapping items, but seems to be a behavioral equivalent of the corresponding factors in Betan et al.
(2005) and Tanzilli et al. (2016), whereas Overwhelmed/Disorganized also contains items with emotional content. If it is true that behaviors are more influenced by the cultural context than basic emotional responses, it would be expected that these two behavioral factors could have more culture-specific expressions, with the same underlying emotional theme. These two factors, Overwhelmed/Disorganized and Special/Overinvolved, both concern issues of the therapeutic frame and boundaries, one in the sense of being forced to set firm limits for the patient, and the other as having difficulties limiting behavior in therapy. One possible interpretation is that this sample of therapists in Sweden are struggling more explicitly with boundary issues compared to the samples of therapists in the earlier studies, indicating some cultural differences. The five remaining factors (Helpless/Inade-

Table 6. Intercorrelations for factor scores between the current factor structure and the Tanzilli et al. (2016) factor structure $(N=\mathbf{2 4 2})$.

Subscales based on Tanzilli et al.'s (2016) factor structure

\begin{tabular}{lcccccccccc}
\hline Factors & $\begin{array}{c}\text { Helpless/ } \\
\text { Inadequate }\end{array}$ & $\begin{array}{c}\text { Overwhelmed// } \\
\text { Disorganized }\end{array}$ & $\begin{array}{c}\text { Positive// } \\
\text { Satisfying }\end{array}$ & $\begin{array}{c}\text { Hostile/ } \\
\text { Angry }\end{array}$ & $\begin{array}{c}\text { Criticized/ } \\
\text { Devalued }\end{array}$ & $\begin{array}{c}\text { Special/ } \\
\text { Overinvolved }\end{array}$ & $\begin{array}{c}\text { Parental// } \\
\text { Protective }\end{array}$ & Sexualized Disengaged \\
\hline 1. Helpless/Inadequate & $\mathbf{0 . 9 5}$ & 0.70 & -0.33 & 0.49 & 0.72 & 0.18 & 0.28 & 0.09 & 0.47 \\
\hline 2. Overwhelmed/Disorganized & 0.53 & $\mathbf{0 . 7 1}$ & -0.22 & 0.55 & 0.58 & 0.19 & 0.14 & 0.34 & 0.40 \\
\hline 3. Hostile/Angry & 0.58 & 0.61 & -0.19 & $\mathbf{0 . 9 3}$ & 0.75 & 0.21 & 0.20 & 0.26 & 0.49 \\
\hline 4. Parental/Protective & 0.25 & 0.36 & 0.49 & 0.15 & 0.20 & 0.42 & $\mathbf{0 . 9 3}$ & 0.10 & 0.07 \\
\hline 5. Disengaged & 0.57 & 0.39 & -0.28 & 0.53 & 0.50 & 0.13 & 0.06 & 0.21 & $\mathbf{0 . 9 5}$ \\
\hline 6. Special/Overinvolved & 0.45 & 0.68 & 0.12 & 0.36 & 0.51 & $\mathbf{0 . 7 2}$ & 0.39 & 0.36 & 0.29 \\
\hline 7. Sexualized & 0.09 & 0.29 & 0.13 & 0.21 & 0.18 & 0.28 & 0.14 & $\mathbf{0 . 9 4}$ & 0.20 \\
\hline
\end{tabular}

Table 7. Comparison between background variables and factor scores/Therapist Response Questionnaire total score.

\begin{tabular}{|c|c|c|c|c|c|c|c|c|c|}
\hline Variable & Method & $\begin{array}{l}\text { TRQ total } \\
\text { score }\end{array}$ & $\begin{array}{c}1 . \\
\text { Helpless/ } \\
\text { Inadequate }\end{array}$ & $\begin{array}{c}2 . \\
\text { Overwhelmed/ } \\
\text { Disorganized }\end{array}$ & $\begin{array}{c}3 . \\
\text { Hostile/ } \\
\text { Angry }\end{array}$ & $\begin{array}{c}4 . \\
\text { Parental/ } \\
\text { Protective }\end{array}$ & $\begin{array}{c}5 . \\
\text { Disengaged }\end{array}$ & $\begin{array}{c}6 . \\
\text { Special/ } \\
\text { Overinvolved }\end{array}$ & $\begin{array}{c}7 . \\
\text { Sexualized }\end{array}$ \\
\hline \multicolumn{10}{|l|}{ Therapist } \\
\hline $\begin{array}{l}\text { Gender } \\
\text { (female vs male) }\end{array}$ & t-test & $\begin{array}{l}\text { Female } \\
<\text { Male }^{*}\end{array}$ & n.s. & $\begin{array}{l}\text { Female } \\
<\text { Male }^{*}\end{array}$ & n.s. & n.s. & n.s. & n.s. & $\begin{array}{l}\text { Female } \\
<\text { Male }^{* *}\end{array}$ \\
\hline Age & Correlation & $r=-.17^{* *}$ & $r=-.20^{* *}$ & n.s. & n.s. & n.s. & n.s. & $r=-.16^{*}$ & n.s. \\
\hline $\begin{array}{l}\text { Basic professional } \\
\text { training }\end{array}$ & ANOVA & n.s. & n.s. & n.s. & n.s. & n.s. & n.s. & n.s. & n.s. \\
\hline $\begin{array}{l}\text { Theoretical orientation } \\
\text { (PDT vs CBT) }\end{array}$ & t-test & $\begin{aligned} & \mathrm{PDT}^{* \mathrm{CBT}^{* * *}}\end{aligned}$ & n.s. & $\begin{aligned} & \mathrm{PDT}^{* *} \\
&>\mathrm{CBT}^{* *}\end{aligned}$ & $\begin{aligned} & \mathrm{PDT}^{* * *} \\
&>\mathrm{CBT}^{* * *}\end{aligned}$ & $\begin{aligned} & \mathrm{PDT}^{* * *} \\
&>\mathrm{CBT}^{* * *}\end{aligned}$ & $\begin{aligned} & \mathrm{PDT}^{* *} \\
&>\mathrm{CBT}^{* *}\end{aligned}$ & n.s. & $\begin{aligned} & \mathrm{PDT}^{* * *} \\
&>\mathrm{CBT}^{* * *}\end{aligned}$ \\
\hline $\begin{array}{l}\text { Experience as } \\
\text { therapist }\end{array}$ & Correlation & $r=-.13^{*}$ & $r=-.14^{*}$ & n.s. & n.s. & n.s. & n.s. & n.s. & n.s. \\
\hline Therapy caseload & Correlation & n.s. & n.s. & n.s. & n.s. & n.s. & n.s. & $r=-.14^{*}$ & n.s. \\
\hline $\begin{array}{l}\text { Setting } \\
\text { (private practice } v s \text { other }\end{array}$ & t-test & $\begin{array}{l}\text { Private } \\
<\text { Other }^{* *}\end{array}$ & $\begin{array}{l}\text { Private } \\
<\text { Other }^{* *}\end{array}$ & $\begin{array}{l}\text { Private } \\
<\text { Other }^{*}\end{array}$ & $\begin{array}{l}\text { Private } \\
<\text { Other }^{*}\end{array}$ & n.s. & n.s. & n.s. & n.s. \\
\hline \multicolumn{10}{|l|}{ Patients } \\
\hline Gender (female $v s$ male) & t-test & n.s. & n.s. & n.s. & n.s. & n.s. & n.s. & n.s. & n.s. \\
\hline Age & Correlation & n.s. & n.s. & n.s. & n.s. & n.s. & n.s. & n.s. & n.s. \\
\hline \multicolumn{10}{|l|}{ Therapies } \\
\hline Length of treatment & Correlation & $r=.18^{* *}$ & n.s. & n.s. & $r=.36^{* *}$ & n.s. & $r=.14^{*}$ & $r=.13^{*}$ & n.s. \\
\hline
\end{tabular}

PDT, Psychodynamic therapy; CBT, Cognitive-behavioral therapy; $* * * \mathrm{P}<.001, * * \mathrm{P}<.01,{ }^{*} \mathrm{P}<.05$; n.s., not significant. 
quate, Hostile/Angry, Parental/Protective, Disengaged and Sexualized) clearly resemble the factors from previous analyses and could have more culturally stable manifestations and effects.

There were, however, differences also between the factor structures in the American and Italian studies respectively, which also could be due to cultural context. This suggests that the cultural context is important to account for when evaluating or comparing therapist reactions, and that the instrument used should be regionally tested.

In other words, we may expect to find both cross-culturally common themes in therapists' reactions, and manifestations of countertransference that are culturally dependent (in the same way as there are idiosyncratic themes in countertransference). However, as there are still no within-culture replications of the factor structure of TRQ, it is difficult to know whether the different results are due to cultural factors or to other circumstances.

Among the factors identified in the American and Italian studies, there were two which were not clearly replicated in the present study: Positive and Criticized/Mistreated. The Hostile/Angry factor in the present study, however, showed a strong correlation to Criticized/Mistreated in Betan et al. (2005) $(r=.77)$ and to Criticized/Devalued in Tanzilli et al. (2016) $(r=.75)$, but an even stronger correlation to Hostile/Angry $(r=.93)$ in the latter study, suggesting that these factors capture a similar phenomenon.

To our surprise, the Positive factor that was identified in both the previous studies did not compare to any even slightly similar factor in the present study. It is notable that TRQ predominantly includes items with negative contents, but that doesn't explain the difference to previous studies. One item from this factor ("I like him/her very much") loaded on the Parental/Protective factor in the present study, and another ("If s/he were not my patient, I could imagine being friends with her") loaded on the Sexualized factor. If this a replicable finding, what does this mean? Could there be something about the Swedish cultural context that makes positive therapist responses to patients (apart from those being part of the Parental/Protective factor) into less of a coherent pattern than in the American and Italian cultural contexts?

Speaking against such a conclusion, however, is that the Swedish studies of the factor structure of the Feeling Word Checklist (FWC) have identified positive factors. Holmqvist et al. (2002), for example, found that their 4four factor solution included a general positive factor, and Lindqvist et al. (2017) identified three positive factors in their 4-factor solution: Engaged, Moved, and Relaxed.

Comparing the TRQ and the FWC shows a number of differences between these two instruments. Especially relevant for the present discussion is that, whereas the items in the TRQ refer mostly to potentially problematic experiences for psychotherapy (resulting in factors with mainly negative contents), the feeling words in different forms of FWC represent a more balanced mix of positive and negative words. A more detailed comparison between these two instruments may nevertheless prove to be important as part of the efforts to develop a more comprehensive taxonomy of therapist reactions and feelings.

The loss of the positive factor could also be attributed to differences in the samples in the different studies. In both Betan et al. (2005) and Tanzilli et al. (2016) more therapists worked in independent settings than in the present study. Therapists working in institutional settings seem to experience more work-related distress and burnout (i.e., fewer positive feelings) in their general work situation (Rupert \& Kent, 2007; Rupert \& Morgan, 2005), and this could secondarily influence the lack of positive countertransference with the particular patient.

The present study identified some groups with higher levels of self-reported countertransference. Male therapists, psychodynamically oriented, younger or less experienced therapists, and therapists in other settings than private practice, all reported more countertransference than female, CBT therapists, older or more experienced therapists, and in private practice. Brody and Farber (1996) also found that less experienced therapists reported higher levels of countertransference in another questionnaire. The reasons for these differences can only be speculated upon and are beyond the scope of the present study, but the results should be taken into consideration in the management of countertransference as well as therapist self-care as higher levels could be connected to risk of burn out or compassion fatigue. Interestingly the caseload for the therapist had no significant effect on countertransference, nor did patient age or gender, or therapist basic education affect TRQ.

The previous studies with TRQ have focused on the patient's contribution to the TRQ results, in the form of personality disorder diagnosis (Betan et al., 2005; Colli et al., 2014; Gazzillo et al., 2015), eating disorder (Satir et al., 2009; Colli et al., 2015) and suicidal behavior (Yaseen et al., 2013). However, the present study has found significant correlations with basic background variables such as therapist age, gender, therapeutic orientation and work-setting, but not patient age or gender. Even if the correlations are rather low, this seems to indicate a contribution to countertransference from basic therapist characteristics but not the patients' corresponding variables. This strengthens Hayes (2004) model that emphasizes the origin of countertransference in the therapist, whereas the situation or the patient's psychopathology might be a trigger.

There are a number of possible reasons for the above differences, for example between CBT and psychodynamically oriented therapists. The patient population could be different and therefore cause different results. The emphasis in the therapeutic training differs concerning countertransference, and the psychodynamically oriented therapists could be more observant of therapist responses, 
and therefore report more countertransference. The length of therapy also seems to contribute to countertransference, and this could also explain some difference between these two schools of therapy.

The difference due to therapist gender is surprising. Why would male therapists experience more countertransference than female therapists? Staczan et al. (2017) found gender-related differences in therapeutic technique where male therapists tended to make more confrontative interventions, that is, interpretations of defense and resistance, whereas female therapists tended to intervene more on an empathic and supportive level. The gender difference is doubtless a complex issue, but one possible understanding of the gender difference in countertransference is that more emphasis on confrontative interventions evokes more reactions in the therapist (and presumably also in the patient) than if the therapist takes a more empathic stance.

Therapists in private practice showed less countertransference than others, although caseload did not seem to matter. These results might indicate the importance of different patient populations, but possibly also the surrounding organization's effect on the individual therapist's work with the particular patient.

\section{Conclusions}

\section{Limitations}

There are at least three important limitations to the present study. First, the sample is rather small, and factor analysis thrives from large samples. Although the number of protocols used in the present EFA $(N=242)$ was larger than in both the American sample $(N=181)$ and Italian subsample $(n=166$; the other half in the total sample was used in a subsequent CFA), it could be objected that the number of protocols is in the lower region. It is possible that a larger number of protocols in all three of the factor analyses of TRQ could result in a convergence in the factor structure (i.e., a larger overlap of items in respective factor or the finding of a positive factor in present study) apart from potential cultural manifestations. The minimum necessary sample size for factor analysis is debatable, and simulation studies suggest a combination of levels of communality in the factor analysis and the ratio of number of variables to the numbers of factors to establish sufficient sample size (MacCallum, Widaman, Zhang, \& Hong, 1999; Mundfrom, Shaw, \& Ke, 2005). According to these recommendations the sample size in the present study ( $N=242$ with wide range of communalities and high overdetermination in number of variables per factor) should be considered sufficient for a stable factor structure.

Second, the sampling procedure was not optimal. The ideal would be to have a random sample of psychotherapists with a good response rate. The therapists, however, were recruited by various channels (four national organi- zations of psychotherapists, Facebook groups, and university trainings) in such a way that no exact response rate could be calculated. On the other hand, Betan et al. (2005) and Tanzilli et al. (2016) reported response rates of $10 \%$ and $29 \%$, respectively, which are also far from ideal. Considering these non-optimal ways of recruiting participants in all three studies, it is quite possible that some difference in results could be due to differences between the samples. For example, diversity in both educational emphasis and temperament among the psychotherapists could create different patterns of reactions to patients.

To evaluate the representativeness of the sample, background variables were compared to available national statistics for psychotherapists in Sweden (Table 1). No significant differences were found, and the sample resembles Swedish psychotherapists in terms of gender $\left(\chi^{2}[1\right.$, $N=241]=.29, \mathrm{P}=.59$ ) and basic education, that is number of psychologists, social workers, nurses and psychiatrists $\left(\chi^{2}[3, N=210]=5.93, \mathrm{P}=.12\right)$.

Also, because countertransference responses may differ depending on the nature of the patients' problems, some differences in results might be due to differences in the patient samples. Because the information about the patients in all three studies is very limited, it is difficult to make any reliable comparisons. It is quite possible, however, that the patients in the present study may represent a partly different population than those in the previous studies. For example, Betan et al. (2005) reported that $49.2 \%$ of their patients had a major depressive disorder, and that $37.6 \%$ had dysthymic disorder; in the present study only $33 \%$ were reported to have a mood disorder. This suggests that mood disorders may have been more common in the American study than in the present one. Further, Tanzilli et al. (2016) recruited a part of their therapists from centers specialized in the treatment of personality disorders, and in terms of DSM-IV personality disorders they reported that of their 332 patients, 18 had a cluster A diagnosis, 71 a cluster B diagnosis, and 58 a cluster $\mathrm{C}$ diagnosis. Even if comorbidity among personality disorder diagnoses is taken into account this is definitely higher than the $12 \%$ reported by the therapists in the present study. If the frequency of mood disorders and/or personality disorder was smaller among the patients in the present study, this might well have influenced the results.

In the same manner, differences between the three studies concerning therapist caseload, work setting, basic professional training, gender, experience as therapist or theoretical orientation could account for differences in factor structure.

A further problem with the sampling procedure is the possibility for the same therapist to contribute with more than one protocol. This is a disadvantage when web-based self-report forms are used, when there is no unique identification of the participants, and when sampling is announced thru various channels. In retrospect we were able 
to identify five likely duplicates when comparing background data for every single protocol. This is a general problem in designs like this, and ideally there should be a system for identification and removal of duplets before the factor analysis is performed. One way of addressing this problem in the future is to instruct the participants that they should only contribute once, even if they were asked via other mediums, or to investigate the technical possibilities for preventing multiple answers. Another way is to include sufficient background data, without compromising anonymity, for identification of duplicates. On the other hand, there are several advantages with the use of web-forms: the removal of one source of errors that could result when the researcher has to manually register data from paper forms, the distribution of the form in a simple and cost efficient way to larger groups (needed for reliable factor analysis), and the possibility of forcing the participant to answer all obligatory questions, which all represent ways to improve the data quality.

A third limitation is that the entire study rests on selfreport data. All kinds of self-report measures have their inherent limits such as cognitive biases and failures to recognize responses that external observers might identify. Also, there has been no validation of the therapist credentials due to our use of a web-form. As Colli and Ferri (2015) suggests, a more reliable design is to add observer or supervisor evaluation to self-report. But then, again, the possibility of psychotherapy research in everyday clinical work is limited.

\section{Future directions}

The above-mentioned limitations point to the need for replications with more clearly representative and larger samples of therapists, as well as the combination of selfreport data with observer ratings. Once the validity of the TRQ is established, however, a number of potential new applications can be envisioned.

One potential use of the TRQ and the factor scores could be as a component in a feedback instrument to the therapist. Systems of feedback from the patient, as for example the Outcome Questionnaire (OQ-45) have been showed to be beneficial to therapy in predicting treatment failure (Lambert, 2013), and feedback from the therapist to him-/herself without the active involvement of the patient could provide the therapist with information potentially useful in managing reactions and notifying the therapist of the hazards of non-managed strong reactions. Through systematic use of feedback instruments the therapist can learn to know his/her idiosyncratic common themes of reactions to patients, as well as reactions evoked in a particular psychotherapy.

In psychotherapy supervision and education, knowledge of the range of common reaction themes could also be useful. The supervisor could readily identify the supervisee's various but common reactions in the therapy and try to make the reaction understandable in the idiosyn- cratic context with a goal of making them containable. To take a concrete example: A therapist working with her first client was bothered by a certain disinterest and distance to the client and blamed herself for this lack of engagement. The supervisor, noting that the description was similar to the disengaged factor in TRQ, conceptualized the reaction in these terms and the discussion in the supervision presented the hypothesis that this disengagement could be evoked by the interaction in the therapy. This led to the identification of a pattern where the patient's need to control different relationships created a situation in the therapy where the patient took complete responsibility for the therapy and the therapist didn't have to work in or before the sessions, creating a feeling of disengagement in the therapist. This recognition helped the therapist to more self-acceptance of her feelings, and made herself more important in the therapy and more focused on the relational aspects of the pattern.

The majority of the factors can be regarded as potentially problematic if actualized, and they are often considered common in the work with patients with personality disorders. By knowing common reaction themes, a therapist can be better prepared to acknowledge both the behavior and emotion, and subsequently manage the reaction.

For further studies and development, it might also be of interest not only to study countertransference at the level of single variables, but also to search for countertransference patterns (i.e., particular combinations of scores on the different TRQ variables, to be identified by cluster analysis) that may characterize particular treatments - and possibly also to search for typical profiles of such patterns that may characterize particular therapists, patients or treatments. For instance, it is possible that certain patterns of countertransference responses are typical of failed or prematurely terminated treatments. To speculate further, the identification of such profiles might not only be useful for therapist feedback but may possibly also open up for new ways of matching patients and therapists in a way that could provide a promising interpersonal mold for successful therapy (Tishby \& Wiseman, 2014).

\section{References}

Betan, E., Heim, A. K., Zittel Conklin, C., \& Westen, D. (2005). Countertransference phenomena and personality pathology in clinical practice: An empirical investigation. The American Journal of Psychiatry, 162, 890-898.

Brody, E. M. \& Farber, B. A. (1996). The effects of therapist experience and diagnosis on countertransference. Psychotherapy: Theory, Research, Practice, Training, 33, 372-380. doi: 10.1037/0033-3204.33.3.372

Colli, A., \& Ferri, M. (2015). Patient personality and therapist countertransference. Current Opinion in Psychiatry, 28, 4656. doi: 10.1097/YCO.0000000000000119

Colli, A., Speranza, A. M., Lingiardi, V., Gentile, D., Nassisi, V., \& Hilsenroth, M. J. (2015). Eating Disorders and Therapist Emotional Responses. Journal of Nervous \& Mental Disease, 
203 (11), 843-849. doi: 10.1097/NMD.0000000000000379

Colli, A., Tanzilli, A., Dimaggio, G., \& Lingiardi, V. (2014). Patient personality and therapist response: An empirical investigation. The American Journal of Psychiatry, 171, 102-108. doi: 10.1176/appi.ajp.2013.13020224

Dahl, H. S. J., Røssberg, J. I., Bøgwald, K. P., Gabbard, G. O., \& Høglend, P. A. (2012). Countertransference feelings in one year of individual therapy: An evaluation of the factor structure in the Feeling Word Checklist-58. Psychotherapy Research, 22, 12-25. doi: 10.1080/10503307.2011.622312

DiStefano, C., Zhu, M., \& Mî̀ndrilă, D. (2009). Understanding and Using Factor Scores: Considerations for the Applied Researcher. Practical Assessment, Research \& Evaluation, 14 (20). Available from: http://pareonline.net/getvn.asp?v $=14 \& \mathrm{n}=20$

Freud, S. (1910). Future prospects of psychoanalytic therapy. The standard edition of the complete works of Sigmund Freud, Volume XXI (pp. 139-151). London: Hogarth Press.

Freud, S. (1912). Recommendations to Physicians Practicing Psycho-Analysis. The standard edition of the complete psychological works of Sigmund Freud, Volume XII (pp. 109120). London: Hogarth Press.

Freud, S. (1915). Observations on Transference-Love (Further Recommendations on the Technique of Psycho-Analysis III). The Standard Edition of the Complete Psychological Works of Sigmund Freud, Volume XII (pp. 157-171). London: Hogarth Press.

Gabbard, G. O. (1995). Countertransference: The Emerging Common Ground. International Journal of Psycho-Analysis, 76, 475-485.

Gabbard, G. O. (2001). A contemporary psychoanalytic model of countertransference. Journal of Clinical Psychology/In Session: Psychotherapy in Practice, 57(8), 983-991.

Gazzillo, F., Lingiardi, V., Del Corno, F., Genova, F., Bornstein, R. F., Gordon, R. M., \& McWilliams, N. (2015). Clinicians' emotional responses and Psychodynamic Diagnostic Manual adult personality disorders: A clinically relevant empirical investigation. Psychotherapy, 52, 238-246. doi: $10.1037 / \mathrm{a} 0038799$

Hayes, J. A. (2004). The inner world of the psychotherapist: A program of research on countertransference. Psychotherapy Research, 14, 21-36.

Hayes, J. A., Gelso, C. J., \& Hummel, A. M. (2011). Managing countertransference. In J. C. Norcross (Ed.), Psychotherapy Relationships That Work: Evidence-Based Responsiveness (pp. 239-258). New York: Oxford University Press.

Heimann, P. (1950). On countertransference. International Journal of Psycho-Analysis, 31, 81-84.

Hoffart, A., \& Friis, S. (2000). Therapists' emotional reactions to anxious inpatients during integrated behavioral-psychodynamic treatment: A psychometric evaluation of a Feeling Word Checklist. Psychotherapy Research, 4, 462-473.

Holmqvist, R. (2001). Patterns of consistency and deviation in therapists' countertransference feelings. Journal of Psychotherapy Practice \& Research, 10(2), 104-116.

Holmqvist, R., \& Armelius, B. A. (1994). Emotional reactions to psychiatric patients. Acta Psychiatrica Scandinavica, 3, 204-209.

Holmqvist, R., Hansjons-Gustafsson, U., \& Gustafsson, J. (2002). Patients' relationship episodes and therapists' feelings. Psychology and Psychotherapy: Theory, Research and Practice, 4, 393-409.

Hu, L. T., \& Bentler, P. M. (1999). Cutoff criteria for fit indexes in covariance structure analysis: Conventional criteria versus new alternatives. Structural Equation Modeling, 6(1), 1-55. doi: 10.1080/10705519909540118

Kanter, J. (2007). Compassion fatigue and secondary traumatization: a second look. Clinical Social Work Journal, 35(4), 289-293.

Lambert, M. J. (2013). Outcome in psychotherapy: the past and important advances. Psychotherapy, 50, 42-51.

Lindqvist, K., Falkenström, F., Sandell, R., Holmqvist, R., Ekeblad, A., \& Thorén, A. (2017). Multilevel exploratory factor analysis of the Feeling Word Checklist-24. Assessment, 24, 907-918. doi: 10.1177/1073191116632336

Lingiardi, V., \& McWilliams, N. (Eds.). (2017). Psychodynamic diagnostic manual (2nd ed.). New York, NY: Guilford Press.

MacCallum, R. C., Widaman, K. F., Zhang, S., \& Hong, S. (1999). Sample size in factor analysis. Psychological Methods, 4, 84-99.

Matsunaga, M. (2010). How to factor-analyze your data right: do's, don'ts, and how-to's. International Journal of Psychological Research, 3, 97-110.

McWilliams, N. (2011). Psychoanalytic diagnosis, second edition: understanding personality structure in the clinical process. New York, NY: Guilford Publications.

Mundfrom, D. J., Shaw, D. G., \& Ke, T. L. (2005). Minimum sample size recommendations for conducting factor analyses. International Journal of Testing, 5, 159-168.

Muthén, L. K., \& Muthén, B. O. (1998-2017). Mplus user's guide. Los Angeles, CA: Muthén \& Muthén.

National Board of Health and Welfare (Socialstyrelsen). (2016). Statistics about licensed psychotherapists in Sweden. Available from: http://www.socialstyrelsen.se/publikationer2016/ 2016-12-4

PDM Task Force. (2006). Psychodynamic Diagnostic Manual. Silver Spring, MD: Alliance of Psychoanalytic Organizations.

Rupert, P. A., \& Kent, J. S. (2007). Gender and work setting differences in career-sustaining behaviors and burnout among professional psychologists. Professional Psychology: Research and Practice, 38(1), 88-96.

Rupert, P. A., \& Morgan, D. J. (2005). Work setting and burnout among professional psychologists. Professional Psychology: Research and Practice, 36, 544-550.

Røssberg, J. I., Hoffart, A., \& Friis, S. (2003). Psychiatric staff members' emotional reactions toward patients. A psychometric evaluation of an extended version of the Feeling Word Checklist (FWC-58). Nordic Journal of Psychiatry, 1, 45-53.

Satir, D. A., Thompson-Brenner, H., Boisseau, C. L., \& Crisafulli, M. A. (2009). Countertransference reactions to adolescents with eating disorders: Relationships to clinician and patient factors. International Journal of Eating Disorders, 42, 511-521. doi: 10.1002/eat.20650

Staczan, P., Schmuecker, R., Koehler, M., Berglar, J., Crameri, A., von Wyl, A., ... Tschuschke, V. (2017). Effects of sex and gender in ten types of psychotherapy. Psychotherapy Research, 27, 74-88. doi: 10.1080/10503307.2015.1072285

Tabachnick, B. G., \& Fidell, L. S. (2014). Using multivariate statistics (6th ed.). Harlow: Pearson Education.

Tanzilli, A., Colli, A., Del Corno, F., \& Lingiardi, V. (2016). Factor structure, reliability, and validity of the Therapist Response Questionnaire. Personality Disorders: Theory, Research, and Treatment, 7(2), 147-158. doi: 10.1037/per 0000146 
Tanzilli, A., Lingiardi, V., \& Hilsenroth, M. (2018). Patient SWAP-200 Personality Dimensions and FFM Traits: do they predict therapist responses? Personality Disorders: Theory, Research, and Treatment, 9(3), 250-262. doi: $10.1037 /$ per0000260

Tishby, O., \& Wiseman, H. (2014). Types of countertransference dynamics: An exploration of their impact on the client-therapist relationship. Psychotherapy Research, 24, 360-375. doi: 10.1080/10503307.2014.893068

Ulberg, R., Falkenberg, A. A., Naerdal, T. B., Johannessen, H., Olsen, J. E., Eide, T. K., ... Dahl, H. S. J. (2013). Countertransference feelings when treating teenagers. A psychometric evaluation of the Feeling Word Checklist-24. American Journal of Psychotherapy, 67(4), 347-358.
Whyte, C. R., Constantopoulos, C., \& Bevans, H. G. (1982). Types of countertransference identified by Q-analysis. British Journal of Medical Psychology, 55, 187-201.

Winnicott, D. W. (1949). Hate in the countertransference. International Journal of Psycho-Analysis, 30, 69-75.

Yaseen, Z. S., Briggs, J., Kopeykina, I., Orchard, K. M., Silberlicht, J., Bhingradia, H., \& Galynker, I. I. (2013). Distinctive emotional responses of clinicians to suicide-attempting patients - a comparative study. BMC Psychiatry 13:230. doi: 10.1186/1471-244X-13-230

Zittel Conklin, C., \& Westen, D. (2003). Therapist response questionnaire. Unpublished manuscript, Department of Psychology, Emory University, Atlanta, GA. 